Instituto Internacional de Investigación y Desarrollo Tecnológico Educativo INDTEC, C.A.

DOI: https://doi.org/10.29394/Scientific.issn.2542-2987.2020.5.18.7.137-156

OAI-PMH: http://www.indteca.com/ojs/index.php/Revista Scientific/oai

Artículo Original / Original Article

\title{
Comprensión del pensamiento educativo de Monseñor Jesús Jáuregui (1848-1905) desde el mundo del texto
}

\author{
Autor: Luis Alberto Velásquez \\ Instituto Universitario de Tecnología del Mar, IUTEMAR \\ losagradoyloprofano.6@gmail.com \\ Boconó, Venezuela \\ https://orcid.org/0000-0002-9295-7568
}

Resumen

El pensamiento de Monseñor Jesús Manuel Jáuregui Moreno, se ha tratado muchas perspectivas epistemológicas y metodológicas, una de ellas es la historiografía, donde el acontecimiento, el testimonio y la huella datada es el principio rector. Ante esta situación se propone el estudio de Mons. Jáuregui desde otro enfoque: la hermenéutica del texto. Aquí, la presente investigación tiene como propósito central comprender su pensamiento educativo comenzando con el mundo del texto. La importancia teórica del trabajo, radica en la posibilidad de hacer un aporte a la recomprensión del pensamiento a luz de la contemporaneidad. En términos metodológicos el trabajo corresponde a una investigación orientada en el paradigma humanointerpretativo, con un tipo de investigación analítica-interpretativa, apoyado en el método Hermenéutico en la concepción de Ricoeur (2004), en la teoría del texto y caracterizado por seguir un diseño procedimental construido a partir la hermenéutica y concretado en la interpretación contenidos en mimesis I, II, III (prefiguración-configuración y refiguración). Para tal fin se interpretaron cuatro obras de orden pedagógicas. En conclusión, el pensamiento educativo de Jáuregui comprendido en el mundo del texto, se le debe considerar: Romántico; Neoescolástico; Multiparadigmático; y Sintagmático.

Palabras clave: pensamiento; interpretación; textos; discurso.

Cómo citar este artículo:

Velásquez, L. (2020). Comprensión del pensamiento educativo de Monseñor Jesús Jáuregui (1848-1905) desde el mundo del texto. Revista Scientific, 5(18), 137-156, e-ISSN: 2542-2987. Recuperado de: https://doi.org/10.29394/Scientific.issn.2542-2987.2020.5.18.7.137-156

Fecha de Recepción: 09-05-2020
Fecha de Aceptación: 21-09-2020
Fecha de Publicación: 05-11-2020 
OAI-PMH: http://www.indteca.com/ojs/index.php/Revista_Scientific/oai

Artículo Original / Original Article

\title{
Understanding the educational thought of Monsignor Jesús Jáuregui $(1848-1905)$ from the world of the text
}

\begin{abstract}
The thought of Monsignor Jesús Manuel Jáuregui Moreno has dealt with many epistemological and methodological perspectives, one of them is historiography, where the event, the testimony and the dated trace is the guiding principle. Faced with this situation, the study of Mons. Jáuregui is proposed from another approach: the hermeneutics of the text. Here, the present research has as its central purpose to understand their educational thinking starting with the world of the text. The theoretical importance of the work lies in the possibility of making a contribution to the re-understanding of thought in light of contemporaneity. In methodological terms the work corresponds to an investigation oriented in the human-interpretive paradigm, with a type of analytical-interpretive investigation, supported by the Hermeneutical method in the conception of Ricoeur (2002), in the theory of the text and characterized by following a Procedural design built from hermeneutics and specified in the interpretation contained in mimesis I, II, III (prefiguration-configuration and refiguration). For this purpose, four pedagogical works were performed. In conclusion, the educational thought of Jáuregui understood in the world of the text, should be considered: Romantic; Neo-scholastic; Multiparadigmatic; and Syntagmatic.
\end{abstract}

Keywords: thought; interpretation; texts; discourse.

\footnotetext{
How to cite this article:

Velásquez, L. (2020). Understanding the educational thought of Monsignor Jesús Jáuregui (1848-1905) from the world of the text. Revista Scientific, 5(18), 137-156, e-ISSN: 2542-2987. Recovered from: https://doi.org/10.29394/Scientific.issn.2542-2987.2020.5.18.7.137-156
}

Date Received: 09-05-2020
Date Acceptance:

21-09-2020
Date Publication:

05-11-2020 


\section{Introducción}

Jesús Manuel Jáuregui Moreno, nació en Niquitao del estado Trujillo, Venezuela, el 28 de septiembre del año 1848. Desde niño sintió el llamado de Dios al servicio de su ministerio, por lo que fue inducido por sus familiares en especial su tío sacerdote a ingresar al Seminario de la ciudad de Mérida. Luego de realizar estudios de filosofía y teología, fue ordenado diácono en el año 1870 y el año siguiente obtuvo la ordenación sacerdotal de manos de Mons. Juan Hilario Bosset (1799-1873). Su vida pastoral las alternó con el estudio y la investigación, obteniendo así, conocimiento sólido en varias disciplinas, que le sirvió para ubicar a la ciencia y la fe en su perfecto lugar. Aprovechó los lugares donde ejerció el sacerdocio para realizar estudios en ciencias físicas y naturales. Sus estudios fueron reconocidos por la comunidad científica del momento, como la Universidad de Boston, la Academia Francesas de Ciencias Naturales y la Sagrada Congregación de Estudios de Roma

Escribió una serie de libros en el orden espiritual, científico y pedagógico que contienen su pensamiento educativo, los cuales fueron utilizados en su colegio como en otros centros de enseñanza de los Andes Venezolanos, formando generaciones de profesionales: teólogos, médicos, literatos, políticos y militares. Al inicio de la revolución restauradora tuvo un altercado y malentendido con el caudillo Cipriano Castro, que al final le valió el exilio. Salió de Venezuela en el año 1899, recorrió varios países como: México, Puerto Rico, Francia, Israel, Roma entre otros. Muere en Roma el 6 de mayo del año 1905.

Para iniciar la descripción de la temática sobre el pensamiento jaureguino, revivimos la voz de Aristóteles (1963a): "la tarea del poeta es describir no lo que ha acontecido, sino lo que podría haber ocurrido, esto es, tanto lo que es posible como probable o necesario" (pág. 21). Es decir, el poeta reflexiona sobre los acontecimientos, buscando la razón última de los mismos, a diferencia del historiador que narra e interpreta el hecho acontecido. En este 
sentido, es importante aclarar y distinguir entre los que escriben literatura aludiendo la percepción y existencia de quien la escribe y los que escriben historia, determinando y radicalizando la conducta del protagonista. En el caso de Aristóteles (1963b): le da una dimensión trascendente a la narración, como un desplegarse hacia una significación refigurante, que no se queda simplemente en una narración de lo datado ejercida por el historiador. Puntualiza Aristóteles (1963c): que "de ahí que la poesía sea más filosófica y de mayor dignidad que la historia, puesto que sus afirmaciones son más bien del tipo universales, mientras que la historia son particulares" (pág. 21).

En la problemática de estos postulados mencionados por Aristóteles (1963d): (acontecimiento datado y el acontecimiento narrado) se pretende abordar el pensamiento educativo de Mons. Jáuregui. En su mayoría las perspectivas de intelectuales como: Mora-García (2003a); Castillo (1999a); y Duque (1999a): se ha interpretado el pensamiento jaureguino en diversos modos que guardan una intención profesional vista desde la historia, teología, filosofía, antropología y la pedagogía. Sin embargo, es relevante señalar que en la actualidad, el intelectual que ha profundizado el pensamiento de Jáuregui es el historiador-filósofo Mora-García (2003b), aplicando la metodología de la historiografía. En este sentido, en cuanto al pensamiento del Levita de los Andes afirma:

Como ha dicho Marc Bloch (1994), "c'est une grande naiveté de prétendre comprendre des hommes sans savoir comment ils se portaient". Los hombres son hijos de su tiempo, para poder conocer su concepción como maestro es necesario comprender al hombre primero, hay que preguntar qué hace y cómo se relaciona con la realidad. De manera que para comprender la mentalidad educativa en Jáuregui debemos preguntar ¿qué hacía? y ¿cómo se relacionaba con la realidad? (pág. 160).

Señalando a Mora-García (2003c): para comprender el pensamiento de Jáuregui hay que conocer su "psiquis" y el "contexto histórico", por lo tanto, 
para conocer a Jáuregui, se debe tener en cuenta la "mentalidad" tema central

de la historiografía francesa en su tercera generación. En concordancia con lo planteado, Ricoeur (2004a), afirma sobre el hombre, la mentalidad y el contexto histórico:

Sobre el problema que preocupa a Marc Bloch, en su capítulo 1 sobre la relación entre "la historia, los hombres y el tiempo". Que el historiador sólo conoce del pasado lo que en él es humano y se deja definir como "ciencia de los hombres en el tiempo"; que el tiempo histórico es a la vez lo continuo y lo desemejante; que la historia debe sustraerse a la obsesión de los orígenes; que el conocimiento del presente es imposible sin el del pasado y presente y recíprocamente. Todos estos temas volverán al primer plano cuando nos preguntemos por los referentes de la historia. Por ahora, sólo nos limitaremos a generalidades epistemológicas que Marc Bloch vincula a sus rápidas reflexiones sobre el objeto $y$, en primer lugar, al estatuto de las nociones de huella y de testimonio (pág. 176).

Se observa en la cita de Ricoeur (2004b): la crítica a la definición de historia de Bloch, reducida a la conducta pasada del hombre, al hecho acontecido en el tiempo, descreditando el presente, sino se apoya en el pasado, porque para Bloch, para entender el pasado debe tener elementos materiales como la huella: vestigio delo acontecido y el testimonio: prueba del pensamiento, línea principal de las mentalidades. No se observa en esta cita la importancia del texto (elemento intencional de la investigación) como mediador entre la intención del autor y la intención del lector, elementos definitorios de la hermenéutica dialogal. En este sentido, se comprende que Mora-García (2003d); y demás autores, abordaron obra y pensamiento de Mons. Jáuregui a partir de la precisión del pasado y de la construcción histórica con las aportaciones de las disciplinas: geografía, antropología, sociología y la psicología.

Frente a esto, Mora-García (2003e): en los estudios sobre el pensamiento de Jáuregui, ha utilizado la historiografía francesa de la tercera 
generación; es decir la historia de las mentalidades de la "Escuela de los Annales". Sobre este punto de vista, Ríos (2009), en relación al tema tratado:

En 1961 Georges Duby (1919-1996) publicaba un artículo intitulado "Histoire des mentalités" en el que reivindicaba la historia de las mentalidades como un objeto de estudio en sí mismo que permitiría enriquecer sustancialmente la historia social y hacía un primer acercamiento teórico. En este texto, el medievalista francés definía la historia de las mentalidades como el estudio de "las respuestas que las distintas sociedades habían dado sucesivamente a la interrogación permanente del hombre a propósito del universo que les engloba y de su destino" y exponía la necesidad de conjugar las aportaciones de la psicología social americana -la cual subrayaba la importancia de las relaciones entre la psicología individual y el medio social en el que el individuo se movía- con las concepciones braudelianas de la larga duración, tal y como Braudel las definía en su célebre artículo aparecido en 1958 en Annales (pág. 100).

Sirve esta afirmación, para demostrar la intención de muchos historiadores que han estudiado a Mons. Jáuregui en ubicarlo en la historia de las mentalidades. En esta perspectiva se deduce para Mora-García (2003f): que no estudió el pensamiento de Jáuregui en la prioridad del texto, tema relevante en la investigación. Con estos comentarios se evidencia que intelectuales antes nombrados han tratado el pensamiento histórico, con epistemología y metodología propia, enmarcada en la visión de la "Escuela de los Annales", han tratado vida, obra y pensamiento de Mons. Jáuregui a partir de la historiografía, ellos no consideran al texto como elemento fundante, mediador ni configurador.

Paralelamente, Mora-García (2003g): plantea una historiografía crítica de los textos de Jáuregui en la que comenta que son punto de referencia para conocer las mentalidades, la historia de los pueblos andinos y en especial la Grita. Por lo tanto, deben ser tratado bajo la precisión de la hermenéutica ontológica, y con esto se descubre la verdadera intención de sus estudios 
conocer la conducta del ser, del hombre llamado Jáuregui. Por lo planteado,

se considera que el autor realiza una historiografía textual, pero buscando el dato, la fecha, la historia, nunca busca el mundo del texto. Con respeto a este comentario, Mora-García (2003h), afirma:

Aunque no es el objetivo del trabajo hacer una historiografía crítica, creemos conveniente hilar unas primeras ideas acerca de los escritos sobre Jáuregui, sobre todo si tenemos en cuenta que la primera compilación es reciente: Dubuc de Isea, L.; Barreto, J.; Porras, B. (1999) (Comp.) Escritos sobre Jáuregui. Alertando que el difícil arte y al mismo tiempo apasionante reto del quehacer historiográfico jaureguino ha sido objeto de escaso análisis por parte de los especialistas. La mayoría de los trabajos escritos sobre el particular, carecen del rigor científico y responden más bien al fruto de la pasión momentánea de la celebración aniversaria de la fecha natalicia (pág. 157).

Se observa en esta afirmación de Mora-García (2003i): la crítica al vacío científico de muchas obras sobre Mons. Jáuregui, pero también el interés de incitar a muchos hacia el estudio y análisis historiográfico sobre el texto educativo del Sócrates Andino. También hace la salvedad de que algunos historiadores 0 intelectuales abordan correctamente la historiografía jaureguiana como es el caso de Castillo (1999b); Luna (1998); y Duque (1999b): con algunos comentarios hacia ellos de halagos historiográficos.

No cabe duda que el trabajo de Mora-García (2003j): en cuanto al tratado del pensamiento de Mons. Jáuregui, sin duda el punto de vista historiográfico ha sido excelente. Sin embargo, hay que hacer la acotación que el objetivo del siguiente trabajo de investigación es comprender el pensamiento educativo de Mons. Jáuregui desde el mundo del texto, donde el epicentro de acción investigativa es el texto que narra el acontecimiento, sirviéndose del acontecimiento datado como referencia secundaria, y tomando como elementos principales mediadores, los símbolos y signos, así como también la comprobación de la temporalidad del texto. 
Continuando con los argumentos sobre la intención de la investigación,

es necesario resaltar la negación de la narración del texto por parte de la historiografía. Ante esto, Ricoeur (2004c), menciona que:

Desgraciadamente, este defecto afecta también a los adversarios del positivismo lógico. Como veremos luego, en el examen de los argumentos "narrativistas", los ejemplos que toma la epistemología, tanto positivista como antipositivista, de los historiadores rara vez alcanzan el nivel de complejidad de las disciplinas históricas actuales. Pero, por heterogéneas que sean las dos corrientes de pensamiento, poseen en común, al menos, además de su negación de la filosofía de la historia, que no nos concierne ahora, la negación del carácter narrativo de la historia tal como se escribe hoy (pág. 170).

Demuestra Ricoeur (2004d): en este apartado que la historiografía niega la narración o el texto, y todo proviene del cambio de objeto o el desplazamiento del objeto de la historia, que en este sentido, pretende relatar el hecho más que comprender el hecho, es decir, su epicentro está en la explicación epistemológica y metodológica del hecho datado.

Ante esta problemática entre la interpretación de texto o paradigma de texto y la historiografía como análisis el hecho histórico social, eidéticamente plantea la problemática entre el hecho histórico y el texto en el tratado del pensamiento educativo de Jáuregui. Ante esta afirmación, surge la siguiente interrogante: ¿Es posible comprender el pensamiento de Mons. Jáuregui en la interpretación de sus textos, tomando como método la hermenéutica del texto?; ¿Es posible dejar a un lado el Dasein o la vía corta y tomar la vía larga o el análisis del lenguaje?. Estas interrogantes se despejarán a lo largo de la investigación cuando se aborde la interpretación del texto comenzando con el proceso mimético.

Después de analizar en algunas obras referidas a la interpretación del texto de Ricoeur (2004e): que se asocia a la comprensión del pensamiento educativo de Mons. Jáuregui plasmado en sus textos, se puede decir, que 
estos poseen un sin fin de signos y símbolos, que aplicando la hermenéutica del texto ayudaran a exteriorizar el significado original de la palabra para reactualizar su sentido, en el mundo sentimental y filosófico del lector.

En cuanto a las obras del prelado andino, Castillo (1999c): escribe que "parte de esa producción intelectual tendía a cumplir una labor divulgativa, llevar conocimientos e ideas a los jóvenes y al pueblo en general. Son entonces los libros de texto para la enseñanza en su Colegio del Sagrado corazón” (pág. 96). Mencionando a Castillo (1999d): el propósito de los textos de Jáuregui, que consistía en difundir los conocimientos aprendidos por el prelado a los estudiantes del Colegio Sagrado corazón de Jesús. Pretendía formarlos en su pensamiento pedagógico, teológico y científico. Según Castillo (1999e): Jáuregui escribió 19 obras, pero en el caso de la investigación, se nombrará cuatro en orden cronológico:

1. Apuntes estadísticos del estado Mérida: Jáuregui (1877a).

2. Tratado de urbanidad para el uso de los seminarios: Jáuregui (1890a).

3. Geometría elemental, para uso de los establecimientos de educación de ambos sexos: Jáuregui (1892a).

4. Introducción a la gramática latina compuesta por Miguel María Candales: Jáuregui (1913a).

A simple vista y reflejándonos en el pensamiento educativo del momento histórico de Mons. Jáuregui se pudiera decir que su pensamiento pudo haber sido influido por algunos elementos (afirmarlo a priori sería una herejía epistemológica). Pero no se puede negar que, si existieron estas líneas de pensamiento precisas que influyeron a Jáuregui, por ejemplo, el pensamiento educativo de Don Bosco; porque lo expresa Castillo (1999f), en la siguiente cita:

Don Bosco respondió luego a Jáuregui ofreciéndole los misioneros solicitados para dentro de cuatro años. Nueva carta 
de Jáuregui a Don Bosco en Majade 1887 y contestación del santo a fines de ese mismo ratificándole la posibilidad de enviar los misioneros en plazo ofrecido. Año. En enero del siguiente año moría Don Bosco y el proyecto de Salesianos para la Grita y Barinas se paraliza (pág. 39).

Se muestra conforme a Castillo (1999g), que existía una profunda relación entre Jáuregui y Don Bosco, de igual forma existe otra evidencia del pensamiento educativo de pastoral-pedagógico de los salesianos en el que:

La llama salesiana se había prendido del Padre Jáuregui inspirado en la obra de don Bosco trató de amoldar su colegio y organización sagrado Corazón de Jesús a los métodos salesianos. Esta inspiración salesiana en la obra jaureguiana la relataba él mismo al delegado Apostólico en una carta de marzo de 1891 (pág. 36).

Se observa de Castillo (1999h): que en el pensamiento de Mons. Jáuregui, hubo influencia del pensamiento de Don Bosco, existía entre ellos vínculos de amistad, y no solo eso, existía afinidad en el carisma salesiano. Es importante profundizar en estas posiciones, porque colabora en la interpretación del texto y a la comprensión del pensamiento educativo del prelado de la Grita, contenida en sus obras.

En este sentido, Mora (2009a), en cuanto al pensamiento y texto de Jesús Manuel Jáuregui, específicamente referido a los manuales que cambiaron la historia de la educación en la Grita:

Destacamos la importancia de tres manuales escolares que cambiaron la historia de la educación en La Grita, (sesión Táchira del Gran Estado los Andes, en el siglo XIX); se trata de los siguientes trabajos escritos por Mons. Jesús Manuel Jáuregui Moreno (1848-1905): (1890) Tratado de Urbanidad para uso de los Seminarios; (1892) Geometría Elemental, para uso de establecimientos de ambos sexos; (1897) Introducción de la Gramática Latina. Estos manuales cambiaron la práctica pedagógica al introducir nuevos enfoques educativos y científicos especialmente influenciados por la tradición pedagógica de Don Bosco y los salesianos. (pág. 251). 
Siguiendo a Mora (2009b): no cabe duda que el pensamiento de Mons. Jáuregui sintió la influencia del pensamiento educativo de Don Bosco. Ante esta afirmación, es necesario resaltar que la presente investigación se justifica o se reviste de importancia porque pretende abordar una alternativa metodológica el estudio del pensamiento juareguino, ya no desde la historiografía, sino la visión metodológica de hermenéutica a través de la interpretación de los textos. En este sentido el contexto histórico en que realizó su obra, se tomara no como lo plantea la historia de las mentalidades, sino como un elemento semiológico.

Esta posición permite interpretarlo y establecer qué relación intelectual y filosófica existía entre la posición de pensamiento y las otras posiciones de pensamiento circundantes y de esta manera realizar un aporte a la recomprensión del discurso pedagógico de Mons. Jáuregui, en el mundo del texto. Pretende mostrar la investigación la aplicación del método de la hermenéutica mediadora textual, así como también la aplicación de los pasos de la mimesis, a los textos de Mons. Jáuregui, con el fin de buscar significados que ayuden a entender su pensamiento, especialmente el educativo.

La investigación en cuanto a lo filosófico-literario, se reviste de importancia porque muestra las características de la dimensión epistemológica donde se abordó la investigación, es decir, la filosofía reflexiva francesa, la fenomenológica-hermenéutica y la antropología filosófica. Tal antropología filosófica, se ve reflejada en la investigación, porque se flexiona sobre el pensamiento contenido en los textos de Jáuregui, dando realce a la existencia y vivencia del sujeto.

Partiendo de la antropología filosofía, es importante dejar claro cuál es el objetivo que persigue la investigación: Comprender el pensamiento educativo de Mons. Jáuregui, es decir, comprender la vivencia y existencia del levita concretado su discurso pedagógico a la luz del siglo XXI, partiendo de la interpretación del mundo del texto, como lo plantea Ricoeur (2004f): en su 
Hermenéutica Dialogal.

\section{Metodología}

La investigación se enmarcó dentro del paradigma humanísticointerpretativo refrendado por Yeaman, Koetting y Nichols (1984): quienes manifiestan que "se centra en el significado de las acciones humanas y de la vida social. Se fundamentan en las condiciones: Comprensión-SignificadoAcción" (pág. 7). Su objetividad se encuentra en el campo de los significados; por lo tanto, corresponde al enfoque cualitativo; se refiere a un estudio hermenéutico, y utilizó un diseño de interpretación de textos.

En el estudio se tomó la Hermenéutica. Con respeto a este método, Ricoeur (2002): añade que "corresponde a la hermenéutica indagar sobre las implicaciones que tiene el devenir del texto para la tarea interpretativa" (pág. 33); es decir, la hermenéutica ilumina el camino procedimental de la comprensión e interpretación de los textos, como recipiente de un pensamiento especifico, pero también como punto de partida para develar la contemporaneidad del lector, asimilándolo con su mundo. En el caso del presente estudio, se pretende interpretar el texto de Mons. Jesús Manuel Jáuregui Moreno, en su texto-discurso-contexto y poder comprender su pensamiento educativo.

El procedimiento para la interpretación de los textos de Mons. Jáuregui y así comprender su pensamiento, se basa en los momentos miméticos, de Ricoeur (2004g), en la que:

Tomo como hilo conductor de este análisis de la mediación entre tiempo y narración la articulación evocada antes e ilustrada ya parcialmente por la interpretación de la poética de Aristóteles entre los tres momentos de la mimesis que llamo: Mimesis I, Mimesis II, Mimesis III. Doy por sabido que mimesis II constituye el eje del análisis; por su función de ruptura, abre el mundo de la composición poética e instituye, como ya he sugerido, la literalidad de la obra literaria. Pero mi tesis es que 
el sentido mismo de la operación de configuración constitutiva de la construcción de la trama resulta de su posición intermedia entre las dos operaciones que yo llamo mimesis I y mimesis III, y que constituyen "el antes" y "el después" de mimesis II. Con esto me propongo mostrar que mimesis II consigue su inteligibilidad de su facultad de mediación, que consiste en conducir del antes al después del texto, transfigurar el antes en después por su poder de configuración (pág. 114).

Explica Ricoeur (2004h): que mediar entre lo escrito en el tiempo y la comprensión interpretación de la misma se utilizará los tres momentos de la mimesis, por ser la más indicada para ocasionar la ruptura de la composición del texto y dejar salir el mundo que encierra para quien lo comprende. Es importe señalar que mimesis II es el lugar donde se configura el sentido del texto, es donde el que comprende une el mundo del texto con su mundo. Sin embargo, no hay que dejar a un lado mimesis I, por ser preconfigurante, donde nace la comprensión con base analítica en la estructura del texto y su inteligibilidad, y la mimesis III, que representar lo último de la comprensión, representa una refiguración del texto, donde el texto se abre y se acopla a las situaciones contemporáneas de los lectores.

En este sentido, para la mimesis como técnica de comprensión, lo más importante y operativo es el texto. La hermenéutica, en cambio, y debido a su amplitud, le interesa la reconstrucción del sentido de obras, autores y lectores. No se limita a colocar mimesis II entre I y III.

Es importante aclarar la importancia de la mimesis (imitación) como procedimiento, ella conlleva a la exactitud de la interpretación, decir el proceso mimético se reviste de rigor científico. Adicionalmente, Castañares (1986): dice que "la exactitud debe consistir en una mimesis: el nombre debe ser la imitación de la esencia mediante las silabas y las letras, el lenguaje es una arte imitativo" (pág. 189). 


\subsection{Procedimiento}

Inspirados en los tres momentos miméticos construidos por Ricoeur (2004i): se procederá a establecer los pasos procedimentales necesarios para interpretar los textos de Mons. Jesús Manuel Jáuregui:

Primer paso: Seleccionar los textos educativos de Mons. Jáuregui a interpretar.

Segundo paso: Aplicar a los textos seleccionado el momento mimético del pre configuración, en la que la composición de la trama tiene sus raíces en la precomprensión del mundo de la acción.

Tercer paso: Aplicar al texto seleccionado el momento mimético de la configuración. Por ser este momento mediador se construirá la trama en la explicación del acontecimiento, de la operación de la configuración y la integración de factores heterogéneos y simbólicos.

Cuarto paso: Aplicar al texto el momento mimético de la refiguración, y el acto de la lectura, el cual es un agente de unión entre configuración y prefiguración, para una significación actual o efectiva del texto. En tanto la actividad lectora supone la fusión de "horizontes", es también el momento de la transformación del texto en obra

Quinto paso: Partiendo de la refiguración, construir un cuerpo significativo actual iniciando por el discurso pedagógico de Mons. Jáuregui, como aporte a su recomprensión.

\section{Resultados}

En la investigación, se seleccionaron cuatro textos relacionados con el pensamiento educativo de Jáuregui a saber, en cuanto a la primera obra seleccionada, Apuntes estadísticos del estado Mérida, manifiesta Jáuregui (1877b): se supone que este texto sirvió de guía didáctica en la práctica pedagógica de Mons. Jáuregui, tanto en el colegio sagrado corazón de Jesús, como en otros centros educativos de primaria, secundaria y universitaria en 
los Andes venezolanos. La vigencia de la obra radica en que, hoy día debe ser considerara como una obra que resume la concepción histórica, geográfica, etnográfica, lingüística, mineralogía y científica del estado Mérida.

Es una obra que, tomándola desde la recomprensión, es pedagógicocientífica, que expresa el alto valor histórico del gentilicio merideño, la descripción geopolítica del estado, muestra las raíces étnicas de los pobladores, muestra la relación cosmogónica de los antepasados y también muestra los rasgos etnohistóricos de la población, pero en la narrativa el autor rescata el sujeto Telúrico (geográfico) el sujeto patrio-pedagógico (evolución histórica de la educación).

La obra en su contexto histórico nace como un elemento cuantificable, pero en una segunda lectura se describe que es más cualificable, ya que muestra elementos del pensamiento del autor como lo son valor a la historia local, valor a la geografía regional, valor por creencias de los indígenas y valor al esfuerzo de la iglesia por evangelizar, contribuyendo con construcción de la identidad andina. En fin, la obra, Apuntes estadísticos del estado Mérida, contiene muchos matices del pensamiento educativo de Mons. Jáuregui en el punto de vista científico, tanto en su forma como en su fondo.

En su segunda obra, Tratado de urbanidad para el uso de los seminarios, de Jáuregui (1890b): se interpreta que adoptó el carisma de los salesianos, y pasó a ser cooperador, y el funcionamiento del colegio fue de inspiración salesiana, por lo tanto, el tratado de urbanidad para uso de seminarios fue un instrumento más para la organización del colegio, pero este se inspiró en los Padres Paules franceses, el manual del Sr. Cardelera y el manual de Manuel Carreño.

El tratado de urbanidad de Jáuregui, lleva en si una actitud afirmativa, que profundiza la reconciliación del hombre entero con su mundo. Jáuregui tenía claro por su condición de teólogo, que el hombre es un ser falible, que constantemente está al acecho del error de la mancha y del pecado. Por tal 
razón, practicó las normas de urbanidad entre los estudiantes de su colegio, como un modo de superación de esta fragilidad, buscando un equilibrio entre el cuerpo, la conciencia y el comportamiento social, que llevó a un significado concreto del cuerpo y el alma y un análisis entre lo objetivo y lo subjetivo.

En la tercera obra seleccionada para interpretar se presenta, Geometría elemental, para uso de los establecimientos de educación de ambos sexos, en la que para Jáuregui (1892b): se puede observar en los significado extraído de la interpretación, sobre la cuadratura del círculo existen varios elementos que están presentes en el pensamiento educativo de Mons. Jáuregui y que subyacen en su textos pero principalmente en "geometría elemental" entre ellos se destacan: evolución del espíritu, unidad entre la vida material y el espíritu, el paso de lo imperfecto a lo perfecto.

La cuadratura del círculo significa la evolución del espíritu humano de la tierra al cielo, mientras que el cuadrado es lo material (la tierra) y el circulo lo espiritual, es decir el cielo. ¿Se pudiera entender esto como una conciliación entre razón (tierra) y fe (cielo) en el pensamiento educativo de Mons. Jáuregui?. Estos términos son comunes en el discurso de Mons. Jáuregui. y concuerdan con positivismo, humanismo y neoescolástica.

En la cuarta obra seleccionada de Jáuregui (1913b), Introducción a la gramática latina compuesta por Miguel María Candales: sin duda alguna que la enseñanza del latín había quedado a traducciones y lecturas en el aula. Mons. Jáuregui en la introducción exhorta a darle más sentido científico a la enseñanza de la gramática latina, dejando claro el sentido pedagógico. Porque impulsa a romper con los viejos paradigmas coloniales de la enseñanza del latín, invita a romper con la didáctica de Nebrija y asumir los nuevos métodos, que ya no solo es repetición y traducción, es ir al significado y su relación con la cultura del momento, el discurso de esta introducción fue una antesala a los nuevos paradigmas lingüísticos de la filosofía Analítica del siglo $\mathrm{XX}$, donde se concibe al lengua como sistema y responde al triangulo semántico: referente- 
significado-significante; como elementos de definición de la gramática latina, superando así el método repetitivo de palabras sin sentido y significados.

\section{Conclusiones}

En las cuatro obras seleccionadas e interpretadas, donde se encuentran tres manuales y un resumen estadístico, se manifiesta que de la interpretación de los textos surge el discurso pedagógico de Mons. Jáuregui hacia el rescate del sujeto patrio, a partir de la enseñanza. El levita de los Andes, busca formar un individuo que ame a su patria, a los próceres, la libertad y a la independencia, enmarcado en el nacionalismo y el patriotismo. Busca rescatar el sujeto místico a través de la enseñanza de elementos místicos, relación Dios y el hombre, relación patria y Dios, basado en la subjetividad religiosa y el respeto por la misma. Busca rescatar el sujeto social desde la enseñanza de los elementos ordenatorios, como manuales, reglamentos y convicciones protocolares, con el fin de formar un ser o individuo que se adapte a las costumbres y quehacer social.

Estas tres dimensiones del sujeto se encuentran inmersas en las estructuras inteligibles de los textos y quedaran en suspenso, para un nuevo encuentro entre el intérprete y el mundo del texto, en la búsqueda de un nuevo significado. En concordancia con lo expuesto, en este encuentro con el mundo del texto de la obra literaria s de Jáuregui se comprendió que su pensamiento se le debe considerar: Romántico; Neoescolástico y multiparadigmáticosintagmático. Será esta investigación punto de partida para futuras estudios sobre Jáuregui, pero también sobre la hermenéutica textual, por representar un emporio de datos que se pueden tomar como referencia al momento de aplicar la técnica de la Mimesis propuesta por Paul Ricoeur.

\section{Referencias}

Aristóteles (1963a,b,c,d). La Poética. Madrid, España: Editorial Aguilar. 
Castañares, W. (1986). El signo: problemas semióticos y filosóficos.

Tesis. Madrid, España: Universidad Complutense de Madrid.

Castillo, L. (1999a,b,c,d,e,f,g,h). Huella imperecedera de Mons. Jáuregui.

En Comisión presidencial para la celebración del sesquicentenario del natalicio de Mons. Dr. Jesús Jáuregui Moreno. Obras completas. Tomo I, ISBN: 980-329-215-3; ISBN: 980-329-216-1. San Cristóbal, Venezuela: Editorial Futuro.

Duque, A. (ed.) (1999a,b). Jáuregui y Silva: contrapunteo epistolar. ISBN: 980-292-800-3. Comisión Presidencial para la celebración del Sesquicentenario del Natalicio de Mons. Dr. Jesús Manuel Jáuregui Moreno. Mérida, Venezuela: Archivo Arquidiocesano de Mérida.

Jáuregui, J. (1913a,b). Introducción a la gramática latina compuesta por Miguel María Candales. En Comisión Presidencial para la celebración del Sesquicentenario del Natalicio de Mons. Dr. Jesús Manuel Jáuregui Moreno. Tomo II, ISBN: 980-329-215-3; ISBN: 980-329-217-X. San Cristóbal, Venezuela: Editorial Futuro.

Jáuregui, J. (1892a,b). Geometría elemental, para uso de los establecimientos de educación de ambos sexos. En Comisión Presidencial para la celebración del Sesquicentenario del Natalicio de Mons. Dr. Jesús Manuel Jáuregui Moreno. Obras Completas, Tomo I. San Cristóbal, estado Táchira: Editorial Futuro.

Jáuregui, J. (1890a,b). Tratado de urbanidad para Uso de los seminarios. En Jáuregui Moreno, J. Obras Completas. Tomo I. San Cristóbal, estado Táchira: Editorial Futuro.

Jáuregui, J. (1877a,b). Apuntes estadísticos del estado Mérida. Cuadernos N. ${ }^{\circ}$ 32. Caracas, Venezuela: Biblioteca Venezolana de Historia.

Luna, H. (1998). Monseñor Jesús Manuel Jáuregui (1848-1905): Contribución al estudio de su biografía y de las relaciones IglesiaEstado en Venezuela. ISBN: 980-200-128-7. Caracas, Venezuela: 
Biblioteca de autores y temas tachirenses.

Mora, J. (2009a,b). Comunidades discursivas de historia de la educación en américa latina, estudio de caso: Venezuela (1998-2008). Tesis doctoral, ISBN: 978-84-692-5921-4. Tarragona, España: Universitat Rovira i Virgili. Recuperado de: http://hdl.handle.net/10803/8945

Mora-García, J. (2003a,b,c,d,e,f,g,h,i,j). J. M. Jáuregui Moreno y su contribución a la historia de la ciencia en la Grita, Sección Táchira del Gran Estado Los Andes (1884-1899). En La Historia de la Ciencia en América Latina. Montalbán, 36. Caracas, Venezuela: Universidad Católica Andrés Bello; Instituto de Investigaciones Históricas.

Ricoeur, P. (2004a,b,c,d,e,f,g,h,i). Tiempo y narración: Configuración del tiempo en el relato histórico. Volumen 1, ISBN: 968-23-1966-8. México: Siglo XXI Editores.

Ricoeur, P. (2002). Del texto a la acción: ensayos de hermenéutica II. ISBN: 968-16-6456-6. México: Fondo de Cultura Económica.

Ríos, M. (2009). De la historia de las mentalidades a la historia cultural: notas sobre el desarrollo de la historiografía en la segunda mitad del siglo XX. Estudios de historia moderna y contemporánea de México, (37), 97-137, e-ISSN: 0185-2620. Recuperado de: http://www.scielo.org.mx/scielo.php?script=sci arttext\&pid=S0185$\underline{26202009000100004}$

Yeaman, A., Koetting, J., \& Nichols, R. (1994). Critical Theory, Cultural Analysis and the Ethics of Educational Technology as Social Responsibility. Educational Technology, 34(2), 5-13, e-ISSN: 00131962. Recovered from: https://www.jstor.org/stable/44428138 


\section{Luis Alberto Velásquez}

e-mail: losagradoyloprofano.6@gmail.com

Nacido en Boconó, Venezuela, el 15 de abril del año

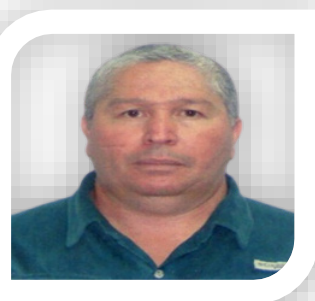
1970. Lcdo. en Filosofía; Lcdo. en Educación; Especialista en Planificación educacional; Doctor en Educación del Instituto Pedagógico Rural "Gervasio Rubio" (IPRGR), Táchira; Docente del Ministerio del Poder Popular para la Educación, Venezuela; Profesor Invitado en Instituto Universitario de Tecnología del Mar (IUTEMAR) de la Fundación La Salle; Docente invitado en la Universidad de los Andes (ULA); Universidad Pedagógica Experimental Libertador (UPEL), Boconó, Trujillo, Venezuela; Docente de Postgrado y Pregrado eventual de la Universidad Pedagógica Experimental Libertador (UPEL); Investigador del PPI A-1.

El contenido de este manuscrito se difunde bajo una Licencia de Creative Commons ReconocimientoNoComercial-Compartirlgual 4.0 Internacional 